\title{
Constraints on Area Variables in Regge Calculus
}

July 6, 2021

\author{
Jarmo Mäkeläa) and Ruth M. Williams ${ }^{b}$ ) \\ a) Department of Physics, University of Jyväskylä, \\ P.O.Box 35, FIN-40351, Jyväskylä, Finland. \\ b) Girton College, Cambridge CB3 0JG, and \\ DAMTP, CMS, Wilberforce Road, Cambridge CB3 0WA, \\ United Kingdom, \\ California Institute of Technology, \\ Pasadena, CA 91125, USA, \\ and \\ CIT-USC Center for Theoretical Physics, \\ University of Southern California, Los Angeles, \\ CA 90089-2535, USA.
}

\begin{abstract}
We describe a general method of obtaining the constraints between area variables in one approach to area Regge calculus, and illustrate it with a simple example. The simplicial complex is the simplest tessellation of the 4-sphere. The number of independent constraints on the variations of the triangle areas is shown to equal the difference between the numbers of triangles and edges, and a general method of choosing these independent constraints is described. The constraints chosen by using our method are shown to imply the Regge equations of motion in our example.
\end{abstract}

Area Regge calculus [1] is a variant of conventional Regge calculus in four dimensions, in which the triangle areas rather than the edge lengths are used as dynamical variables [2]. Although for a single 4-simplex, the numbers of edges $N_{1}$ and of triangles $N_{2}$ are equal, this is not true for a general simplicial complex, where we usually have $N_{2}>N_{1}$. The counting of degrees of freedom in a 
discrete theory is never completely straightforward, and given the ambiguity in the number of true variables, there are two attitudes one can take in area Regge calculus.

Firstly, one can take the area variables as the fundamental ones, with edges entering only as a tool for calculating volumes and deficit angles. (Recall [2] that for a generic 4-simplex, it is possible to solve uniquely for the edge-lengths in terms of the areas.) This approach has been investigated in detail in [2] and it has been shown [3] that although the equations of motion constrain all the deficit angles to be zero, the theory has non-trivial dynamical content.

Secondly, one can regard some of the areas as redundant variables and aim to reduce their number to the number of edge lengths in the simplicial complex. This possibility has been considered in detail by Mäkelä 迎. In order to recover the conventional approach to simplicial gravity where the edge lengths are real physical quantities, it is necessary to impose the condition that a given edge has the same length in whichever 4-simplex that length is calculated. (Strangely enough, this is by no means automatic - see [2].) This leads to a large number of constraints: for each edge, there is a constraint for each pair of 4-simplices sharing that edge. A total of $N_{2}-N_{1}$ of these constraints will be independent, but it is not easy to give a universal rule to say how to choose them. Mäkelä [4 has shown that if the variations of the constraints are added in with Lagrange multipliers to the variation of the Regge action expressed in terms of area variables, then the usual Regge calculus equations of motion are recovered.

The purpose of this letter is to propose a general method of finding an independent set of constraints and to illustrate it with a specific model.

The key point of the method is the observation that when 24 -simplices are put together along a common tetrahedron, the number of edges is 14 , whereas the number of triangles is 16 . Thus the difference between the numbers of triangles and edges is 2 , from which it follows that we need 2 constraints on the triangle areas to ensure that the edge-lengths in the tetrahedron have consistent values, independent of the 4 -simplex in which they are calculated. It appears that we can choose any 2 of the edges of the common tetrahedron and that the corresponding constraints are always independent.

We now proceed further. Denoting the 24 -simplices already discussed above by $\sigma_{1}$ and $\sigma_{2}$, we now consider a new 4 -simplex $\sigma_{3}$, which shares a common tetrahedron with $\sigma_{2}$. This new 4-simplex contains a number of new triangles, which is always greater than, or the same as, the number of new edges (the difference is usually, but not always, equal to 2 ). We now pick a number, equal to this difference, of edges from the tetrahedron shared between $\sigma_{2}$ and $\sigma_{3}$, and write down the corresponding constraints. 
This process is repeated. We first count the difference between the number of triangles and the number of edges appearing when a new 4-simplex is considered, and write the corresponding number of constraints, chosen as described, until eventually the total number of constraints equals the difference between the number of triangles and the number of edges for the whole complex.

We now describe a simple model to which we can apply this procedure. The simplicial complex chosen is $\alpha_{5}$, the tessellation of $S^{4}$ by the surface of a five-simplex, which is the complete graph on six points, labelled $0,1, \ldots, 5$. The numbers of simplices of each dimension are as follows: $N_{0}=6, N_{1}=15, N_{2}=$ $20, N_{3}=15, N_{4}=6$. This means that we expect the number of independent constraints to be $N_{2}-N_{1}=5$.

For simplicity, we start with all the edge lengths set equal to 1 , and the triangle areas to $\sqrt{3} / 2$, and then allow small variations. We label the variations in squared-edge-length by $\delta s_{i j}$ and in area by $\delta A_{i j k}$, where $i, j, k \ldots$ label vertices. Since these variations are small, we keep only linear terms.

To show how the constraints are derived, we give one example. Solving for the variation of the edge $s_{01}$ in the 4 -simplex with vertices 01234 , we obtain

$$
\begin{gathered}
(\sqrt{3} / 2) \delta s_{01}=2 \delta A_{012}+2 \delta A_{013}+2 \delta A_{014}+2 \delta A_{234}-\delta A_{023}-\delta A_{024}-\delta A_{034} \\
-\delta A_{123}-\delta A_{124}-\delta A_{134} .
\end{gathered}
$$

Similarly, in simplex 01235, we have

$$
\begin{gathered}
(\sqrt{3} / 2) \delta s_{01}=2 \delta A_{012}+2 \\
2 A_{013}+2 \delta A_{015}+2 \delta A_{235}-\delta A_{023}-\delta A_{025}-\delta A_{035} \\
-\delta A_{123}-\delta A_{125}-\delta A_{135}
\end{gathered}
$$

Since we require $\delta s_{01}$ to be independent of the simplex in which it is calculated, we obtain our first constraint by equating the expressions in (1) and (2), giving

$$
\begin{aligned}
& 2 \delta A_{014}+2 \delta A_{234}-\delta A_{024}-\delta A_{034}-\delta A_{124}-\delta A_{134} \\
= & 2 \delta A_{015}+2 \delta A_{235}-\delta A_{025}-\delta A_{035}-\delta A_{125}-\delta A_{135} .
\end{aligned}
$$

We denote this constraint by $[01,45]$. In general, $[i j, k l]$ stands for the constraint that $\delta s_{i j}$ be the same in the two 4 -simplices which have all vertices except $k$ and all except $l$. Clearly this makes sense only if neither $i$ nor $j$ is equal to $k$ or $l$.

Each edge is shared by four 4-simplices, so taking the expressions for $\delta s$ two at a time, we obtain 6 constraints for each edge. There are 15 edges, so the total number of constraints is 90 . However this can be reduced to half immediately by noticing that, for each edge, only 3 of the possible differences between pairs 
are independent. (In general, if $n$ 4-simplices meet on an edge, then by equating the values of the square of that edge-length in successive 4-simplices, we see that there will always be a total of $n-1$ independent constraints there.) This can be expressed symbolically by

$$
[i j, k l]=[i j, k m]-[i j, l m],
$$

where $m$ is the label of one of the remaining vertices. Note that there is an ambiguity in the sign of each term, since the actual constraint is $[i j, k l]=0$.

By inspection of the constraints obtained, it can be seen that

$$
[i j, k l]=[m n, k l],
$$

where $(i j k l m n)$ is one permutation of (012345), and that

$$
[i j, k l]=[i m, k l]-[j m, k l]
$$

where again $m$ is the label of one of the remaining vertices.

The number of relations of the type given in (4) is 60 , there are 45 of type (5) and 60 of type (6), so clearly they are not all independent and we cannot subtract their total number from the number of constraints.

To understand the origin of the constraints of type (5), we need to consider the action of the symmetry group of the simplicial complex, which is $S_{6}$, the permutation group on 6 objects. For example, consider the relation

$$
[01,45]=[23,45] .
$$

Now the permutations in $S_{6}$ which exchange the edges 01 and 23 are (0213), (0312), (02)(13) and (03)(12). Acting on triangles, they leave invariant the sets $(014,234),(015,235),(012,013,023,123),(024,034,124,134)$ and $(025,035$, $125,135)$. These are precisely the sets of triangles which leave the constraints $[01,45]$ and $[23,45]$ unchanged.

For the relations of type (6), the symmetries are more subtle; there is an elaborate matching between the terms which enter the constraints, and this is a feature of the particular simplicial complex used.

A convenient way of considering the constraints is as follows. Since there are 20 triangles, we regard the variations of areas (the $\delta A_{i j k}$ ) as normalised basis vectors of a 20-dimensional vector space, $V_{20}$. Each of the 90 constraints corresponds to a vector $\mathbf{C}_{i}, i=1, \ldots, 90$, in this space. Clearly there cannot be more than 20 linearly independent $\mathbf{C}_{i}$, and in fact we have shown by straightforward but tedious calculation that only 5 of them are linearly independent. 
This is precisely the number $N_{2}-N_{1}$ of linearly independent constraints that we expect.

To see why this number has to be 5 , consider a linear extension of $V_{15}$, the 15 dimensional vector space with basis the normalised vectors corresponding to the variations of the edge lengths in the simplicial complex. We denote the vector corresponding to the variation in edge $i j$ by $\mathbf{v}_{\mathbf{i j}}$, and that corresponding to the variation in the area of triangle $i j k$ by $\mathbf{V}_{\mathbf{i j k}}$. We define a linear homomorphism $\phi$ from $V_{15}$ into $V_{20}$ by

$$
\phi: \mathbf{v}_{\mathbf{i j}} \rightarrow \mathbf{V}_{\mathbf{i j k}}+\mathbf{V}_{\mathbf{i j l} \mathbf{l}}+\mathbf{V}_{\mathbf{i j m}}+\mathbf{V}_{\mathbf{i j n}},
$$

where (ijklmn) is some permutation of (012345). This mapping is $S_{6}$-linear:

$$
\phi(g \mathbf{v})=g(\phi \mathbf{v}) \text { for all } g \in S_{6},
$$

and it is also injective, as can be seen by checking that its kernel is trivial. The vector space structure of $V_{15}$ is obviously inherited by its image in $V_{20}$, so we have a 15 -dimensional subspace of $V_{20}$, which we denote by $\operatorname{Im}(\phi)$. This is an $S_{6^{-}}$ invariant subspace of $V_{20}$. It is then a simple matter to check that every vector $\mathbf{C}_{\mathbf{i}}$ is orthogonal to all the vectors spanning $\operatorname{Im}(\phi)$. For example, look back at (2) and represent it by $\mathbf{C}_{\boldsymbol{1}}$. Then $\mathbf{C}_{\mathbf{1}}$ is orthogonal to $\mathbf{V}_{\mathbf{0 1 2}}+\mathbf{V}_{\mathbf{0 1 3}}+\mathbf{V}_{\mathbf{0 1 4}}+\mathbf{V}_{\mathbf{0 1 5}}$ and so on. Thus the vectors $\mathbf{C}_{\mathbf{i}}$ span a nontrivial subspace of $V_{20}$ orthogonal to $\operatorname{Im}(\phi)$, which must therefore have dimension at most 5 . It must also be an $S_{6}$-invariant subspace of $V_{20}$ (note that the scalar product is invariant under $S_{6}$ ) and so must have dimension precisely 5 (the representation theory of $S_{6}$ implies that the orthogonal complement subspace is irreducible).

We are now ready to apply our general method of choosing independent constraints to our simplicial complex, $\alpha_{5}$. Since it has only 6 vertices, it is convenient to denote the 4 -simplex from which vertex $k$ is missing by $\sigma_{k}$.

Consider first the 4 -simplices $\sigma_{0}$ and $\sigma_{5}$, which meet on the tetrahedron with vertices (1234). We pick the edges (12),(13), say, from this tetrahedron, and write the corresponding constraints, which, in the notation introduced above, are $[12,05]$ and $[13,05]$. It is easily checked that these are independent.

Next we consider the 4-simplex $\sigma_{1}$. This meets $\sigma_{0}$ on the tetrahedron (2345) and $\sigma_{5}$ on the tetrahedron (0234). It brings in one new edge (05) and 3 new triangles $(025),(035),(045)$. We choose 2 edges, $(23),(24)$ say, from the common tetrahedra, and write down 2 constraints for them, $[23,01]$ and $[24,01]$ say. These are again independent of each other, and also independent of the constraints already introduced.

So far we have constrained the variables in $\sigma_{0}, \sigma_{1}$ and $\sigma_{5}$, and we have 4 constraints out of the 5 that we need. Let us now consider $\sigma_{2}$, which meets $\sigma_{1}$ 
on the tetrahedron (0345). We pick just one edge, (03) say, with the constraint $[03,12]$. It is straightforward to check that this is independent of the others. One possible set of constraints is then

$$
\text { [12, 05], [13, 05], [23, 01], [24, 01], [03, 12]. }
$$

Obviously the choice is not unique.

Having obtained an independent set of constraints, we can then check that, as in [4, where all the constraints are added with Lagrange multipliers to the action, we obtain the usual Regge equations of motion. Suppose we take the set of constraints listed in (10), and add them to the variation of the action, with Lagrange multipliers $\lambda_{i}$, giving [4]

$$
\begin{gathered}
\delta S=-\frac{1}{8 \pi} \frac{1}{3 !} \delta A_{i j k} \phi_{i j k} \\
+\lambda_{1}[12,05]+\lambda_{2}[13,05]+\lambda_{3}[23,01]+\lambda_{4}[24,01]+\lambda_{5}[03,12],
\end{gathered}
$$

where $\phi_{i j k}$ is the deficit angle at the triangle with the corresponding labels. Setting $\delta S=0$ and equating coefficients of each $\delta A$, we obtain expressions for the deficit angles. For example,

$$
\begin{gathered}
\phi_{012}=8 \pi\left(2 \lambda_{1}-\lambda_{2}\right), \\
\phi_{013}=8 \pi\left(-\lambda_{1}+2 \lambda_{2}+2 \lambda_{5}\right), \\
\phi_{014}=8 \pi\left(-\lambda_{1}-\lambda_{2}-\lambda_{5}\right), \\
\phi_{015}=8 \pi\left(-\lambda_{5}\right) .
\end{gathered}
$$

Now the equation of motion obtained by varying with respect to $s_{01}$ involves the sum over triangles of $\left(\partial A_{i j k} / \partial s_{01}\right) \phi_{i j k}$. Since the lattice we are using is totally symmetric, the $\partial A / \partial s$ is a fixed number, independent of both the triangle and of the edge, and the sum we want just involves multiplying equations (15)-(18) by that number and adding them. (Note that the deficit angles listed are for all the triangles which contain $s_{01}$.) It is clear that we immediately obtain the usual equation of motion

$$
\frac{\partial A_{i j k}}{\partial s_{01}} \phi_{i j k}=0 .
$$

Similarly for variations with repect to all the other $s_{i j}$.

In this letter we have described a method of picking a set of independent constraints between variations of area variables in a general simplicial lattice. Using this method we obtained a set of independent constrants in a simple 
example and showed that they imply the ususal Regge equations of motion. This understanding of the general constraint structure of classical area Regge calculus should pave the way for an investigation of the corresponding quantum theory.

\section{Acknowledgements}

The authors thank Jan Saxl for help with group theory. One of them (RMW) is grateful for hospitality at the Theory Division at CERN, where this work was completed. The work was supported in part by the UK Particle Physics and Astronomy Research Council.

\section{References}

[1] C. Rovelli, Phys. Rev. D 48, 2702 (1993).

[2] J. W. Barrett, M. Roček and R. M. Williams, Class. Quantum Grav. 16, 1373 (1999).

[3] T. Regge and R. M. Williams, J. Math. Phys. 41, 3964 (2000).

[4] J. Mäkelä, "Variation of area variables in Regge calculus", gr-qc/9801022. 